\title{
Approximating the Probability Distribution of OFDM Symbol Errors
}

\author{
Alan Clark, Peter Smith \& Desmond Taylor \\ Department of Electrical and Computer Engineering \\ University of Canterbury \\ Christchurch, New Zealand \\ Email: a.clark@elec.canterbury.ac.nz
}

\begin{abstract}
Given an $N$ subcarrier orthogonal frequency division multiplexing (OFDM) system transmitting over a slow fading Rayleigh channel, the distribution of $b$, the number of received symbol errors, is Poisson binomial. Hence, $\left(\begin{array}{c}N \\ b\end{array}\right)$ terms are required to calculate each probability for $b=0,1, \ldots, N$. When $N$ is large, as in most OFDM systems, the Poisson binomial distribution is often approximated by the Poisson distribution. We show that, for large $N$, the total variation distance between the approximation and the true distribution is lower and upper bounded by random variables with fully known probability density functions. The bounds on the total variation distance indicate that the distribution of OFDM symbol errors is well approximated by a Poisson distribution.
\end{abstract}

\section{INTRODUCTION}

Given an orthogonal frequency division multiplexing (OFDM) system transmitting $N$ subcarriers over a Rayleigh fading channel, a block of $N$ subcarrier symbols is sent simultaneously. The probability of $b \in\{0,1, \ldots, N\}$ of the $N$ symbols being incorrectly estimated at the receiver is of interest. Knowledge of the probability distribution of the number of errors affords better error correction code design and analysis, since any code of minimum distance $d_{m i n}$ corrects $b<\frac{d_{\min }}{2}$ errors. This knowledge is applicable in adapting OFDM to achieve a desired error rate [1].

For a slowly fading Rayleigh channel we show that the probability of $b$ OFDM symbol errors is well approximated by the Poisson distribution. Since each subchannel has a different time varying gain, the probability of error for each OFDM symbol is time varying. Thus, for fixed time $t$, the distribution of the number of errors is equivalent to the distribution of the sum $B$ of a number of independent Bernoulli random variables, $X_{1}, X_{2}, \ldots, X_{N}$, with respective success probabilities $p_{1}, p_{2}, \ldots, p_{N}$, known commonly as the Poisson binomial distribution. The Poisson binomial distribution is well approximated by the Poisson distribution, with a known upper bound [2] on the total variation distance. The total variation distance upper bound is a random variable, for which we derive an asymptotic probability density function (pdf) for a large number of subcarriers $N$. Knowledge of this pdf allows us to construct confidence intervals on the accuracy of the approximation.
In the next section we introduce necessary notation, assumptions and preliminary results concerning OFDM systems, multipath channels and error rates. Section III outlines the derivation of the pdf of the upper bound on the total variation distance. Section IV shows simulated probability distributions for an OFDM system, as well as the distribution of the upper bound on total variation distance from the Poisson approximation. Finally, Section V provides some conclusions.

\section{Preliminaries}

\section{A. OFDM System Notation}

We assume readers are familiar with OFDM systems [3], and merely summarise notation. During the $n^{\text {th }}$ discrete time interval an $N$ subcarrier OFDM system transmits data symbol $S_{n, k}$ on the $k^{\text {th }}$ subcarrier. We refer to the superposition of $N$ subcarrier symbols as the $n^{\text {th }}$ OFDM block. We assume here that $S_{n, k} \in\left\{-\sqrt{E_{0}}, \sqrt{E_{0}}\right\}$, that is a binary phase shift keying (BPSK) symbol with energy $E_{0}$.

We assume perfect receiver synchronisation in time and frequency, ideal channel state information, and sufficient guard interval for inter block interference to be negligible. Then, during the $n^{\text {th }}$ time interval the received signal can be written

$$
R_{n, k}=H_{n, k} S_{n, k}+N_{n, k},
$$

for the $k^{\text {th }}$ subcarrier, where $N_{n, k}$ is a zero mean complex Gaussian random variable with variance $\frac{N_{0}}{2}$ per dimension, and $H_{n, k}$ is the $k^{\text {th }}$ complex subchannel gain for time interval $n$. The receiver then estimates the transmitted symbol as the closest point to $\hat{S}_{n, k}=\frac{R_{n, k}}{H_{n, k}}=S_{n, k}+\frac{N_{n, k}}{H_{n, k}}$. The instantaneous SNR for the $k^{\text {th }}$ subcarrier, in the $n^{\text {th }}$ time interval is denoted $\gamma_{n, k}=\left|H_{n, k}\right|^{2} \frac{E_{0}}{N_{0}}$.

\section{B. Multipath Channel Notation}

We denote the time-varying channel frequency response over the entire OFDM bandwidth as $H(t, f)$, and make the usual wide sense stationary and uncorrelated scattering assumptions [4]. Furthermore, we assume a constant, time independent channel ensemble mean of zero, and an exponential delay power profile with mean delay $\tau_{D}$. We denote the expectation of some function $X_{k}=g\left(H\left(t, f_{k}\right)\right)$ at fixed frequency $f_{k}$ as $\bar{X}_{k}=\mathbb{E}\left[g\left(H\left(t, f_{k}\right)\right)\right]$. 
The OFDM subchannel gains are the channel response at frequencies $\left\{f_{k}=f_{c}+\left(k-\frac{N}{2}\right) \Delta f\right\}$, for $k=1,2, \ldots, N$, where $\Delta f$ is the OFDM subcarrier separation and $f_{c}$ is the carrier frequency. The OFDM block then has period $T=$ $\frac{N}{\Delta f}$. Assuming a slowly fading channel, the gain on the $k^{\text {th }}$ subchannel during the $n^{\text {th }}$ time interval $n T<t \leq(n+1) T$ is approximately constant, and denoted $H_{n, k}$. Furthermore, we may write [4]

$$
H_{n_{1}, k_{1}}=y_{n_{1}, k_{1}}^{(1)}+j y_{n_{1}, k_{1}}^{(2)}, \quad H_{n_{2}, k_{2}}=y_{n_{2}, k_{2}}^{(1)}+j y_{n_{2}, k_{2}}^{(2)},
$$

where $y_{n_{1}, k_{1}}^{(1)}, y_{n_{1}, k_{1}}^{(2)}, y_{n_{2}, k_{2}}^{(1)}$ and $y_{n_{2}, k_{2}}^{(2)}$ are identically distributed, zero mean Gaussian random variables. These have the correlation properties

$$
\begin{aligned}
\mathbb{E}\left[\left(y_{n_{1}, k_{1}}^{(1)}\right)^{2}\right] & =\frac{1}{2}, \quad \mathbb{E}\left[y_{n_{1}, k_{1}}^{(1)} \cdot y_{n_{1}, k_{1}}^{(2)}\right]=0, \\
\mathbb{E}\left[y_{n_{1}, k_{1}}^{(1)} \cdot y_{n_{2}, k_{2}}^{(1)}\right] & =\mathbb{E}\left[y_{n_{1}, k_{1}}^{(2)} \cdot y_{n_{2}, k_{2}}^{(2)}\right] \\
& =\frac{1}{2} \frac{J_{0}\left(2 \pi f_{d}\left|n_{1}-n_{2}\right|\right)}{1+\left(2 \pi \Delta f \Delta k \tau_{D}\right)^{2}}, \\
\mathbb{E}\left[y_{n_{1}, k_{1}}^{(1)} \cdot y_{n_{2}, k_{2}}^{(2)}\right] & =-\mathbb{E}\left[y_{n_{1}, k_{1}}^{(1)} \cdot y_{n_{2}, k_{2}}^{(2)}\right] \\
& =-\left(2 \pi \Delta f \Delta k \tau_{D}\right) \mathbb{E}\left[y_{n_{1}, k_{1}}^{(1)} \cdot y_{n_{2}, k_{2}}^{(1)}\right],
\end{aligned}
$$

where $\Delta k=\left|k_{2}-k_{1}\right|, f_{d}$ is Doppler frequency and $J_{0}(\cdot)$ is the zero order Bessel function of the first kind. We observe from (3) that the marginal distribution of each channel gain envelope $\left|H_{n, k}\right|$ follows a Rayleigh distribution. Finally, we denote the arithmetic mean over all subchannels of some function $X_{n}=g(H(n T, f))$, at time interval $n$, as $X_{n, a v}=$ $\frac{1}{N} \sum_{k=1}^{N} g\left(H\left(n T, f_{k}\right)\right)$.

\section{OFDM Symbol Error Rates}

The probability $P_{n, k}$ of the $k^{\text {th }}$ subcarrier symbol received during the $n^{\text {th }}$ time interval being incorrectly estimated, and the squared probability of error may be written as

$$
\begin{aligned}
& P_{n, k}=\mathrm{Q}\left(\sqrt{\frac{2 E_{0}}{N_{0}}} \sqrt{\left[y_{n, k}^{(1)}\right]^{2}+\left[y_{n, k}^{(2)}\right]^{2}}\right) \triangleq p\left(y_{n, k}^{(1)}, y_{n, k}^{(2)}\right), \\
& P_{n, k}^{2}=\mathrm{Q}^{2}\left(\sqrt{\frac{2 E_{0}}{N_{0}}} \sqrt{\left[y_{n, k}^{(1)}\right]^{2}+\left[y_{n, k}^{(2)}\right]^{2}}\right) \triangleq q\left(y_{n, k}^{(1)}, y_{n, k}^{(2)}\right),
\end{aligned}
$$

where $Q($.$) is the well known Gaussian Q-function. The$ Hermite ranks [5] $\varphi(p)$ and $\varphi(q)$, of $p\left(y_{n, k}^{(1)}, y_{n, k}^{(2)}\right)$ and $q\left(y_{n, k}^{(1)}, y_{n, k}^{(2)}\right)$ respectively, are greater than or equal to two (Appendix I).

For time interval $n$, we denote the arithmetic mean probability of symbol error across all $N$ subcarriers as $P_{n, a v}=$ $\frac{1}{N} \sum_{k=1}^{N} P_{n, k}$, and the mean squared probability of error as $P_{n, a v}^{2}=\frac{1}{N} \sum_{k=1}^{N} P_{n, k}^{2}$. For any subcarrier $k$ we denote the expected probability of error as

$$
\bar{P}_{k}=\mathbb{E}\left[P_{n, k}\right]=\int_{0}^{\infty} \mathrm{Q}\left(\sqrt{2 \gamma_{n, k}}\right) f_{\gamma}\left(\gamma_{n, k}\right) d \gamma_{n, k},
$$

where $f_{\gamma}\left(\gamma_{n, k}\right)$ is the pdf of the instantaneous SNR, $\gamma_{n, k}$. For a Rayleigh distributed channel envelope, it is well known [6] that $\gamma_{n, k}$ follows an exponential probability density function, $f_{\gamma}(x)={\overline{\gamma_{k}}}^{-1} \exp \left(\frac{x}{\bar{\gamma}_{k}}\right)$. Assuming that all subchannel gains have equal time averaged subcarrier SNR $\overline{\gamma_{k}}=\overline{\gamma_{0}}=\frac{E_{0}}{N_{0}}$, it may be shown [6] that

$$
\overline{P_{k}}=\overline{P_{0}}=\frac{1}{2}\left[1-\sqrt{\frac{\overline{\gamma_{0}}}{1+\overline{\gamma_{0}}}}\right], k=1,2, \ldots, N .
$$

\section{ACCURACY OF POISSON Approximation}

\section{A. Distribution of Subcarrier Average Error Rate}

We apply the following theorem from [5] to show that the average subcarrier error rate $P_{n, a v}$ and average squared error rate $P_{n, a v}^{2}$ are asymptotically Gaussian distributed in time.

Theorem 1 (Arcones) Let $\left\{\mathbf{X}_{i}\right\}_{i=1}^{\infty}$ be a stationary meanzero Gaussian sequence of vectors in $\mathbb{R}^{d}$. Set $\mathbf{X}_{i}=$ $\left(X_{i}^{(1)}, \ldots, X_{i}^{(d)}\right)$. Let $f$ be a function on $\mathbb{R}^{d}$ with Hermite rank, [5], $\varphi$ such that $1 \leq \varphi<\infty$. Define

$$
r^{(p, q)}(k)=E\left[X_{m}^{(p)} X_{m+k}^{(q)}\right],
$$

for $k \in \mathbb{Z}$, where $m$ is any number large enough that $m \geq 1$ and $m+k \geq 1$. Suppose that

$$
\sum_{k=-\infty}^{\infty}\left|r^{(p, q)}(k)\right|^{\varphi}<\infty
$$

for all $1 \leq p \leq d$ and $1 \leq q \leq d$. Then

$$
\frac{1}{\sqrt{n}} \sum_{j=1}^{n}\left(f\left(\mathbf{X}_{j}\right)-E\left[f\left(\mathbf{X}_{j}\right)\right]\right) \stackrel{d}{\longrightarrow} \mathcal{N}\left(0, \sigma^{2}\right)
$$

where ' $\stackrel{d}{\longrightarrow}$ ' denotes, 'convergence in distribution', and

$$
\begin{aligned}
& \sigma^{2}=E\left[\left(f\left(X_{1}\right)-E\left[f\left(X_{1}\right)\right]\right)^{2}\right] \\
& +2 \sum_{k=1}^{\infty} E\left[\left(f\left(X_{1}\right)-E\left[f\left(X_{1}\right)\right]\right)\left(f\left(X_{1+k}\right)-E\left[f\left(X_{1+k}\right)\right]\right)\right]
\end{aligned}
$$

We show that the theorem applies to an OFDM system with a large number of subcarriers, transmitting over Rayleigh fading channels, since the subchannel gain at time $n$ is a Gaussian random variable in $\mathbb{R}^{2},(2)$. Furthermore, the subcarrier error probability $P_{n, k}$ and the subcarrier squared error probability $P_{n, k}^{2}$ are defined as functions of $\mathbb{R}^{2}$ valued Gaussian random variables, as seen in (4). It is then shown that the distribution of both the average subcarrier error $P_{n, a v}$ and squared subcarrier error $P_{n, a v}^{2}$ approach Gaussian distributions.

At time interval $n$ consider two OFDM subchannel gains $H_{n, k_{1}}=y_{n, k_{1}}^{(1)}+j y_{n, k_{1}}^{(2)}$ and $H_{n, k_{2}}=y_{n, k_{2}}^{(1)}+j y_{n, k_{2}}^{(2)}$, with properties given in (3). Letting $k_{1}, k_{2} \in\{1,2, \ldots, N\}$ and 
$\Delta k=\left|k_{2}-k_{1}\right|$, we may write

$$
\begin{aligned}
& \sum_{\Delta k=-\infty}^{\infty}\left|\mathbb{E}\left[y_{n, k_{1}}^{(1)} y_{n, k_{1}}^{(1)}\right]\right|^{\varphi(p)} \\
= & \sum_{\Delta k=-\infty}^{\infty}\left|\mathbb{E}\left[y_{n, k_{1}}^{(2)} y_{n, k_{1}}^{(2)}\right]\right|^{\varphi(p)} \\
= & \frac{1}{2^{\varphi(p)}}\left[1+\sum_{\Delta k=-\infty, \Delta k \neq 0}^{\infty}\left|\frac{1}{1+\left(2 \pi \Delta f \Delta k \tau_{D}\right)^{2}}\right|^{\varphi(p)}\right],
\end{aligned}
$$

which is finite since $\varphi(p) \geq 2$, as shown in Appendix I. Furthermore, the summation

$$
\begin{aligned}
& \sum_{\Delta k=-\infty}^{\infty}\left|\mathbb{E}\left[y_{n, k_{1}}^{(1)} y_{n, k_{1}}^{(2)}\right]\right|^{\varphi(p)} \\
= & \sum_{\Delta k=-\infty}^{\infty}\left|\mathbb{E}\left[y_{n, k_{1}}^{(2)} y_{n, k_{1}}^{(1)}\right]\right|^{\varphi(p)} \\
= & \frac{1}{2^{\varphi(p)}} \sum_{\Delta k=-\infty, \Delta k \neq 0}^{\infty}\left|\frac{2 \pi \Delta f \Delta k \tau_{D}}{1+\left(2 \pi \Delta f \Delta k \tau_{D}\right)^{2}}\right|^{\varphi(p)}
\end{aligned}
$$

is also convergent, since $\varphi(p) \geq 2$. Thus requirement (8) of Theorem 1 is satisfied, and the distribution of mean subcarrier error rates $P_{n, a v}$ approaches a Gaussian distribution, so that

$$
\begin{aligned}
& \frac{1}{\sqrt{N}} \sum_{k=1}^{N}\left\{p\left(y_{n, k}^{(1)}, y_{n, k}^{(2)}\right)-\mathbb{E}\left[p\left(y_{n, k}^{(1)}, y_{n, k}^{(2)}\right)\right]\right\} \\
& \stackrel{d}{\longrightarrow} \mathcal{N}\left(0, N \operatorname{var}\left(P_{n, a v}\right)\right) .
\end{aligned}
$$

Therefore, for large $N$ we may state

$$
P_{n, a v} \stackrel{d}{\sim} \mathcal{N}\left(\overline{P_{0}}, \operatorname{var}\left(P_{n, a v}\right)\right),
$$

where ' $\stackrel{d}{\sim}$ ' denotes approximated in distribution, and in (13) and (14)

$$
\begin{aligned}
& N \operatorname{var}\left(P_{n, a v}\right) \\
& =\mathbb{E}\left[\left(p\left(y_{n, 1}^{(1)}, y_{n, 1}^{(2)}\right)-\mathbb{E}\left[p\left(y_{n, 1}^{(1)}, y_{n, 1}^{(2)}\right)\right]\right)^{2}\right] \\
& +2 \sum_{k=2}^{\infty} \mathbb{E}_{t}\left[\left(p\left(y_{n, 1}^{(1)}, y_{n, 1}^{(2)}\right)-\mathbb{E}\left[p\left(y_{n, 1}^{(1)}, y_{n, 1}^{(2)}\right)\right]\right)\right. \\
& \left.\quad\left(p\left(y_{n, k}^{(1)}, y_{n, k}^{(2)}\right)-\mathbb{E}\left[p\left(y_{n, k}^{(1)}, y_{n, k}^{(2)}\right)\right]\right)\right] \\
& =\overline{P_{0}^{2}}-\left[\overline{P_{0}}\right]^{2}+2 \sum_{k=2}^{\infty}\left\{\mathbb{E}\left[P_{n, 1} . P_{n, k}\right]-\left[\overline{P_{0}}\right]^{2}\right\} .
\end{aligned}
$$

The quantities $\left.\overline{P_{0}^{2}}, \overline{P_{0}}\right]^{2}$ and $\mathbb{E}\left[P_{n, 1} . P_{n, k}\right]$ are calculable using the results of Appendix II.

Since $\varphi(q) \geq 2$ (Appendix I), similar evaluations of cross correlations sums to (11) and (12) hold for the squared subcarrier error rate, and we may use Theorem 1 to show that the average subcarrier squared error rate also approaches a Gaussian distribution. That is,

$$
\begin{aligned}
& \frac{1}{\sqrt{N}} \sum_{k=1}^{N}\left\{p^{2}\left(y_{n, k}^{(1)}, y_{n, k}^{(2)}\right)-\mathbb{E}\left[p^{2}\left(y_{n, k}^{(1)}, y_{n, k}^{(2)}\right)\right]\right\} \\
& \stackrel{d}{\longrightarrow} \mathcal{N}\left(0, N \operatorname{var}\left(P_{n, a v}^{2}\right)\right) .
\end{aligned}
$$

Therefore, for large $N$ we may state

$$
P_{n, a v}^{2}(n) \stackrel{d}{\sim} \mathcal{N}\left(\overline{P_{0}^{2}}, \operatorname{var}\left(P_{n, a v}^{2}\right)\right),
$$

where in (16) and (17)

$$
\begin{aligned}
& N \operatorname{var}\left(P_{n, a v}^{2}\right) \\
&= \mathbb{E}\left[\left(p^{2}\left(y_{n, 1}^{(1)}, y_{n, 1}^{(2)}\right)-\mathbb{E}\left[p^{2}\left(y_{n, 1}^{(1)}, y_{n, 1}^{(2)}\right)\right]\right)^{2}\right] \\
&+2 \sum_{k=2}^{\infty} \mathbb{E}_{t}\left[\left(p^{2}\left(y_{n, 1}^{(1)}, y_{n, 1}^{(2)}\right)-\mathbb{E}\left[p^{2}\left(y_{n, 1}^{(1)}, y_{n, 1}^{(2)}\right)\right]\right)\right. \\
&\left.\cdot\left(p^{2}\left(y_{n, k}^{(1)}, y_{n, k}^{(2)}\right)-\mathbb{E}\left[p^{2}\left(y_{n, k}^{(1)}, y_{n, k}^{(2)}\right)\right]\right)\right] \\
&=\overline{P_{0}^{4}}-\left[\overline{P_{0}^{2}}\right]^{2}+2 \sum_{k=2}^{\infty}\left\{\mathbb{E}\left[P_{n, 1}^{2} . P_{n, k}^{2}\right]-\left[\overline{P_{0}^{2}}\right]^{2}\right\} .
\end{aligned}
$$

The quantities $\overline{P_{0}^{4}}$ and $\mathbb{E}\left[P_{n, 1}^{2} . P_{n, k}^{2}\right]$ are readily calculable, as outlined in Appendix II.

\section{B. Accuracy of Approximations to Poisson Binomial}

Our concern is the approximation of the distribution of the number of errors in an OFDM system with a large number of subcarriers, say $N \geq 128$, transmitting over a Rayleigh fading channel. Such systems have already been proposed and commissioned, [7]. For a given OFDM block $n$ the probability of there being $b$ symbol errors follows a Poisson binomial distribution, with density function denoted $\mathcal{L}_{n}(b)$, and probabilities $P_{n, 1}, P_{n, 2}, \ldots, P_{n, N}$ defined by (4).

Calculation of the Poisson binomial distribution requires the summation of $\left(\begin{array}{c}N \\ b\end{array}\right)$ terms, for $b=0,1, \ldots, N$. This has motivated work on approximating the Poisson binomial distribution with the Poisson distribution [2], [8]-[11]. The total variation distance between an approximating distribution, $\mathcal{F}_{n}(b)$, and the Poisson binomial distribution, is defined [2] as

$$
d\left\{\mathcal{L}_{n}(b), \mathcal{F}_{n}(b)\right\}=\frac{1}{2} \sum_{a=0}^{\infty}\left|\mathcal{L}_{n}(a)-\mathcal{F}_{n}(a)\right| .
$$

For the $n^{\text {th }}$ block, we use the Poisson distribution $\mathcal{P}_{\lambda_{n}}(b)$ as an approximation, where $\lambda_{n}=N P_{n, a v}=\sum_{k=1}^{N} P_{n, k}$. It is proven in [2] that this approximation has total variation distance bounded by

$$
\begin{aligned}
& d\left\{\mathcal{L}_{n}(b), \mathcal{P}_{\lambda_{n}}(b)\right\} \geq \frac{1}{32} \min \left\{\frac{1}{\lambda_{n}}, 1\right\} \sum_{k=1}^{N} P_{n, k}^{2}, \\
& d\left\{\mathcal{L}_{n}(b), \mathcal{P}_{\lambda_{n}}(b)\right\} \leq \frac{1-e^{-\lambda_{n}}}{\lambda_{n}} \sum_{k=1}^{N} P_{n, k}^{2} .
\end{aligned}
$$


For each block we approximate the probability of there being $b$ errors with a Poisson distribution. We then consider the limit, as $N \rightarrow \infty$, of the total variation distance bounds. It is readily shown from (20) that

$$
\begin{aligned}
& \lim _{N \rightarrow \infty} d\left\{\mathcal{L}_{n}(b), \mathcal{P}_{\lambda_{n}}(b)\right\} \geq \frac{1}{32} \frac{\sum_{k=1}^{N} P_{n, k}^{2}}{\sum_{k=1}^{N} P_{n, k}}=\frac{1}{32} \frac{P_{n, a v}^{2}}{P_{n, a v}}=L_{n}, \\
& \lim _{N \rightarrow \infty} d\left\{\mathcal{L}_{n}(b), \mathcal{P}_{\lambda_{n}}(b)\right\} \leq \frac{\sum_{k=1}^{N} P_{n, k}^{2}}{\sum_{k=1}^{N} P_{n, k}}=\frac{P_{n, a v}^{2}}{P_{n, a v}}=U_{n} .
\end{aligned}
$$

The total variation distance between the Poisson and the Poisson binomial distribution is a random variable in time, that is along OFDM blocks, due to the fading nature of the Rayleigh channel. Therefore, the bounds (20) and (21) on total variational distance are also random variables. Recall that the limiting distributions of $P_{n, a v}$ and $P_{n, a v}^{2}$ are Gaussian. Then the distributions of the limits in (21) are given by the distributions of the ratios of two correlated Gaussian random variables. The distribution of such ratios is derived in [12] and we then have $U_{n} \stackrel{d}{\longrightarrow} f_{U}(x)$, where

$$
f_{U}(x)=\frac{c_{2} \exp \left(-\frac{1}{2}\left[a^{2}+b^{2}\right]\right)}{\pi\left[c_{2}^{2}+\left(x-c_{1}\right)^{2}\right]}\left[1+\sqrt{\frac{\pi}{2}} D \frac{\operatorname{erf}\left(\frac{D}{\sqrt{2}}\right)}{\exp \left(-\frac{D^{2}}{2}\right)}\right] .
$$

Variables $D, c_{1}, c_{2}, a$ and $b$ are defined as

$$
\begin{aligned}
& a=\frac{1}{\sqrt{1-\rho^{2}\left(P, P^{2}\right)}}\left[\frac{\mathbb{E}\left[P_{n, a v}^{2}\right]}{\sqrt{\operatorname{var}\left(P_{n, a v}^{2}\right)}}-\frac{\rho\left(P, P^{2}\right) \mathbb{E}\left[P_{n, a v}\right]}{\sqrt{\operatorname{var}\left(P_{n, a v}\right)}}\right] \\
& D=\frac{b c_{2}+a\left(x-c_{1}\right)}{\sqrt{c_{2}^{2}+\left(x-c_{1}\right)^{2}}}, \quad c_{1}=\rho\left(P, P^{2}\right) \sqrt{\frac{\operatorname{var}\left(P_{n, a v}^{2}\right)}{\operatorname{var}\left(P_{n, a v}\right)}}, \\
& b=\frac{\mathbb{E}\left[P_{n, a v}\right]}{\sqrt{\operatorname{var}\left(P_{n, a v}\right)}}, \quad c_{2}=\sqrt{1-\rho^{2}\left(P, P^{2}\right)} \sqrt{\frac{\operatorname{var}\left(P_{n, a v}^{2}\right)}{\operatorname{var}\left(P_{n, a v}\right)}},
\end{aligned}
$$

where $\rho\left(P, P^{2}\right)$ is the correlation coefficient between $P_{n, a v}$ and $P_{n, a v}^{2}$, which is easily calculable from the correlation between them (Appendix II). A similar result may be shown for the limiting distribution of $L_{n}$. It is observed from (14)(18) and (21) that as the number of subcarriers $N$ increases, the variance in the limiting distributions of the bounds on total variation distance does not increase. Furthermore, for fixed mean SNR, the mean of these limiting distributions remains constant.

\section{Simulations}

We consider a 1024 subcarrier OFDM system transmitting over a Rayleigh fading channel with an exponential power delay profile and maximum excess delay of $50 \mathrm{~ns}$. We assume the OFDM system occupies a $320 \mathrm{MHz}$ bandwidth with carrier frequency $5.1 \mathrm{GHz}$ and receiver velocity of $15 \mathrm{~m} / \mathrm{s}$. Due to the large number of subcarriers, calculation of the exact probability of $b$ errors for $3 \leq b \leq 1022$ is infeasible.

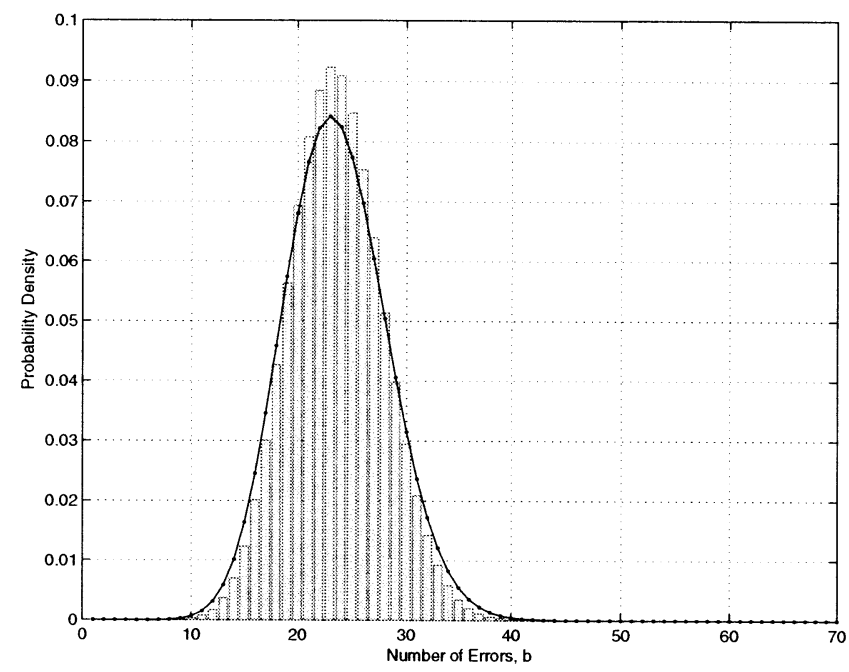

Fig. 1. Simulated Error Rate Distribution (bars) and Poisson Approximation (solid line) for one Channel Realization.

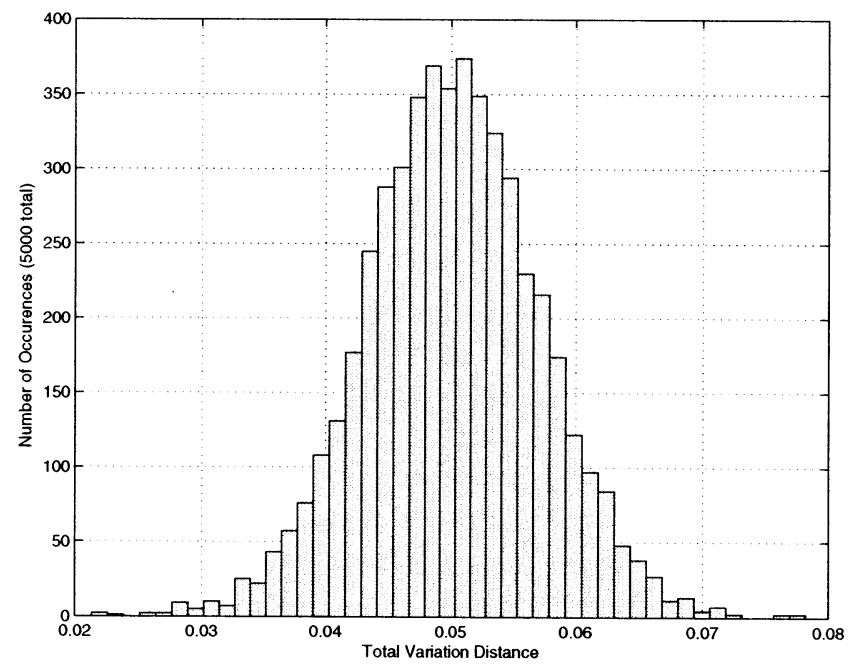

Fig. 2. Simulated Total Variation Distance Between Poisson and Poisson Binomial Distributions

We consider transmission over 5000 consecutive simulated fading channel realizations. For each channel realization we simulate transmission of $10^{7}$ blocks, and thus estimate the distribution of errors for that channel realization. We then calculate the total variation distance between the estimated distribution and the Poisson approximation, as well as bounds on total variation distance (21) for each realization.

In Figure 1 we plot the simulated distribution of errors and the Poisson approximation for a single channel realization. It is observed that the Poisson distribution is a good approximation to the simulated distribution of errors, with total variation distance of 0.0458 . We display the distribution of the total variation distance between the simulated distribution of errors and the Poisson approximation, for all 5000 realizations, in Figure 2, and plot the expected distribution of the upper bound on the total variation distance in Figure 3.

It is observed from Figure 3 that the pdf of (22) closely 


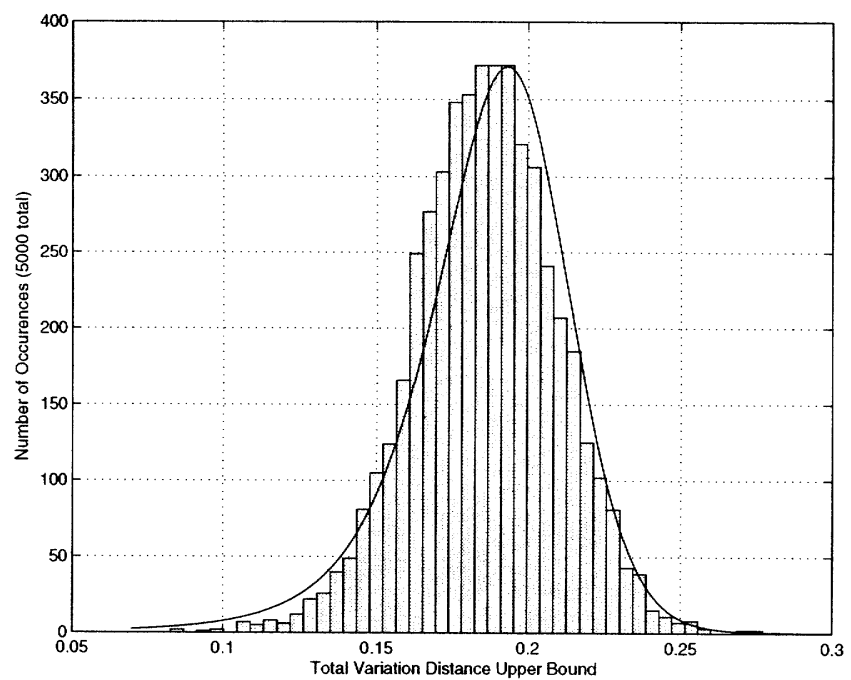

Fig. 3. Upper Bound on the Total Variation Distance, Simulated (histogram) and Approximating Density Function (solid line, scaled for 5000 trials)

approximates the density of the simulated upper bound on total variation distance. Similar results are readily obtained for the lower bound. As $N$ increases, the approximation becomes tighter.

\section{Concluding Remarks}

For an OFDM system transmitting BPSK over a Rayleigh fading channel we have considered Poisson distribution approximation of the distribution of the number of symbol errors per received block. The total variation distance is a random variable in time due to the fading nature of the channel, and is bounded above and below by random variables whose probability distribution has been derived. The upper bound on total variation distance between the true distribution and the Poisson approximation does not increase as the number of subcarriers increases.

The Poisson distribution appears to be a good approximation to the distribution of the number of OFDM symbol errors per block, for fading, frequency selective channel realizations. The results obtained enable us to construct confidence intervals on the approximation error when estimating the probability of a given number of OFDM errors per received block for a Rayleigh fading channel. The analysis may be readily extended to other symbol constellations, such as quadrature amplitude modulation.

\section{APPENDIX I}

\section{HERMITE RANK OF ERROR FUNCTIONS}

Given the gain for the $k^{\text {th }}$ subcarrier over the $n^{\text {th }}$ time interval, $H_{n, k}=y_{n, k}^{(1)}+j y_{n, k}^{(2)}$, where $y^{(1)}$ and $y^{(2)}$ are Gaussian random variables with properties given in (3), we may express the probability of incorrectly estimating the transmitted BPSK symbol as (4) for given symbol energy $E_{0}$ and noise power spectral density $N_{0}$.

The Hermite rank $\varphi(f)$ of a measurable function $f: \mathbf{X} \rightarrow$ $\mathbb{R}$ for the mean zero, independent, identically distributed (iid)
Gaussian vector $\mathbf{X}=\left\{X_{1}, \ldots, X_{d}\right\} \in \mathbb{R}^{d}$, where $f$ has finite second moment, may be defined [5] as

$$
\begin{aligned}
& \inf \{\varphi(f): \exists \text { polynomial } P \text { of degree } \varphi(f) \text { with, } \\
& \left.\quad \mathbb{E}\left[(f(\mathbf{X})-\mathbb{E}[f(\mathbf{X})]) \cdot P\left(X^{(1)}, \ldots, X^{(d)}\right)\right] \neq 0\right\} .
\end{aligned}
$$

We show that the Hermite rank, $\varphi(p)$, of $p\left(y_{n, k}^{(1)}, y_{n, k}^{(2)}\right)$ is greater than two, by showing that it is neither zero nor unity. Firstly consider a polynomial on $\left\{y_{n, k}^{(1)}, y_{n, k}^{(2)}\right\}$ of degree zero, that is $f_{0}\left(y_{n, k}^{(1)}, y_{n, k}^{(2)}\right)=A_{0}$, for some constant $A_{0} \in \mathbb{R}$. We can then write

$$
\begin{aligned}
& \mathbb{E}\left[\left\{P_{n, k}-\mathbb{E}\left[P_{n, k}\right]\right\} f_{0}\left(y_{n, k}^{(1)}, y_{n, k}^{(2)}\right)\right] \\
= & \mathbb{E}\left[\left\{P_{n, k}-\mathbb{E}\left[P_{n, k}\right]\right\} A_{0}\right] \\
= & 0, \forall A_{0},
\end{aligned}
$$

since $\mathbb{E}\left[\mathbb{E}\left[P_{n, k}\right]\right]=\mathbb{E}\left[P_{n, k}\right]$, and thus $\varphi(p) \neq 0$. Secondly consider a first degree polynomial on $\left\{y_{n, k}^{(1)}, y_{n, k}^{(2)}\right\}$, denoted $f_{1}\left(y_{n, k}^{(1)}, y_{n, k}^{(2)}\right)=A_{1,1} y_{n, k}^{(1)}+A_{1,2} y_{n, k}^{(2)}+A_{0}$. Then

$$
\begin{aligned}
& \mathbb{E}\left[\left\{P_{n, k}-\mathbb{E}\left[P_{n, k}\right]\right\} f_{1}\left(y_{n, k}^{(1)}, y_{n, k}^{(2)}\right)\right] \\
= & \mathbb{E}_{t}\left[A_{1,1} y_{n, k}^{(1)} P_{n, k}+A_{1,2} y_{n, k}^{(2)} P_{n, k}+A_{0} P_{n, k}\right. \\
& \left.-\mathbb{E}\left[P_{n, k}\right] A_{1,1} y_{n, k}^{(1)}-\mathbb{E}\left[P_{n, k}\right] A_{1,2} y_{n, k}^{(2)}-\mathbb{E}\left[P_{n, k}\right] A_{0}\right] \\
= & \mathbb{E}\left[A_{1,1} y_{n, k}^{(1)} P_{n, k}\right]+\mathbb{E}\left[A_{1,2} y_{n, k}^{(2)} P_{n, k}\right],
\end{aligned}
$$

since $y_{n, k}^{(1)}$ and $y_{n, k}^{(2)}$ are zero mean, iid Gaussian random variables. We denote the bivariate Gaussian probability density function of $y_{n, k}^{(1)}$ and $y_{n, k}^{(2)}$ as $f\left(y^{(1)}, y^{(2)}\right)$. Considering the first term of the final line of (26) and using the Gaussian Q-function representation of [13] we may write

$$
\begin{aligned}
& \mathbb{E}\left[A_{1,1} y_{n, k}^{(1)} P_{n, k}\right] \\
= & A_{1,1} \int_{-\infty}^{\infty} \int_{-\infty}^{\infty} y^{(1)} Q\left(2 \sqrt{\left[y^{(1)}\right]^{2}+\left[y^{(2)}\right]^{2}} \sqrt{\frac{E_{0}}{N_{0}}}\right) \\
& f\left(y^{(1)}, y^{(2)}\right) d y^{(1)} d y^{(2)}, \\
= & \frac{A_{1,1}}{\pi} \int_{0}^{\frac{\pi}{2}} \int_{-\infty}^{\infty} \exp \left(-\frac{2 E_{0}\left[y^{(2)}\right]^{2}}{N_{0} \sin ^{2} \phi}\right) \\
& \cdot\left\{\int_{-\infty}^{\infty} y^{(1)} \exp \left(-\frac{2 E_{0}\left[y^{(1)}\right]^{2}}{N_{0} \sin ^{2} \phi}\right) f\left(y^{(1)}, y^{(2)}\right) d y^{(1)}\right\} \\
& \cdot d y^{(2)} d \phi
\end{aligned}
$$$$
=0, \forall A_{1,1} \text {. }
$$ 
This follows because the integrand within the parentheses $\{\ldots\}$ on the penultimate line of (27) is the product of an odd function and two even functions of $y^{(1)}$. We can similarly show that $\mathbb{E}\left[A_{1,1} y_{n, k}^{(1)} P_{n, k}\right]=0$, for all $A_{1,2}$. Substituting these results into (26) we deduce that $\varphi(p)$ is neither zero nor one, and therefore at least 2.

Using a similar approach it is readily shown that the Hermite rank $\varphi(q)$ of the average squared probability of error $P_{n, a v}^{2}$ is at least 2 .

\section{APPENDIX II}

\section{SUbCARRIER ERROR FUNCTION CORRELATIONS}

At time $n$ the correlation between the squared values of the channel envelopes, $\left|H_{n, k_{1}}\right|^{2}$ and $\left|H_{n, k_{2}}\right|^{2}$, is [4]

$$
\rho_{H}(\Delta k)=\frac{1}{\sqrt{1+\left(2 \pi \Delta f \Delta k \tau_{D}\right)^{2}}} .
$$

The corresponding channel SNR's $\gamma_{n, k_{1}}$ and $\gamma_{n, k_{2}}$ have a bivariate exponential distribution which may be written [14] as

$$
\begin{aligned}
& f_{\gamma, \gamma}\left(\gamma_{n, k_{1}}, \gamma_{n, k_{2}}\right) \\
= & \kappa \exp \left(-\alpha\left[\gamma_{n, k_{1}}+\gamma_{n, k_{2}}\right]\right) I_{0}\left(\theta \sqrt{\gamma_{n, k_{1}} \gamma_{n, k_{2}}}\right),
\end{aligned}
$$

with

$$
\begin{aligned}
\kappa & =\frac{1}{{\overline{\gamma_{0}}}^{2}\left(1-\rho_{H}(\Delta k)^{2}\right)}, \quad \alpha=\frac{1}{\overline{\gamma_{0}}\left(1-\rho_{H}(\Delta k)^{2}\right)} \\
\theta & =\frac{2 \rho_{H}(\Delta k)}{\overline{\gamma_{0}}\left(1-\rho_{H}(\Delta k)^{2}\right)} .
\end{aligned}
$$

The correlation between the error probabilities on channels $k_{1}$ and $k_{2}$ may then be written as a rapidly convergent series, allowing simple calculation [15]. That is,

$$
\mathbb{E}\left[P_{n, k_{1}} P_{n, k_{2}}\right]=\kappa \sum_{i=0}^{\infty} \frac{\theta^{2 i}}{4^{i} \alpha^{2(i+1)}} C_{i}^{2}, \text { for } k_{1} \neq k_{2},
$$

where

$$
C_{i}=\left(\frac{1-\mu_{\alpha}}{2}\right)^{i+1} \sum_{n=0}^{i}\left(\begin{array}{c}
i+n \\
n
\end{array}\right)\left(\frac{1+\mu_{\alpha}}{2}\right)^{n}
$$

and $\mu_{\alpha}=\sqrt{\frac{1}{1+\alpha}}$.

Furthermore, in the special case of $k_{1}=k_{2}$, we use a result from [16] to write

$$
\overline{P_{k}^{2}}=\overline{P_{0}^{2}}=\frac{1}{4}-\frac{\arctan \left(\sqrt{{\overline{\gamma_{0}}}^{-1}+1}\right)}{\pi \sqrt{{\overline{\gamma_{0}}}^{-1}+1}} .
$$

Similarly the correlation between the squared error rates on two channels $k_{1}$ and $k_{2}$ may also be written as the rapidly convergent series [15],

$$
\mathbb{E}\left[P_{n, k_{1}}^{2} P_{n, k_{2}}^{2}\right]=\sum_{i=0}^{\infty} \frac{\theta^{2 i} \kappa}{4^{i}} D_{i}^{2} \text { for } k_{1} \neq k_{2},
$$

where $D_{i}$ is the readily calculable finite range integral

$$
\frac{1}{\pi^{2}} \int_{0}^{\frac{\pi}{2}} \int_{0}^{\frac{\pi}{2}}\left[\frac{\sin ^{2} \phi \sin ^{2} \vartheta}{\beta \sin ^{2} \vartheta \sin ^{2} \phi+\sin ^{2} \vartheta+\sin ^{2} \phi}\right]^{i+1} d \phi d \vartheta .
$$

In the special case of $k_{1}=k_{2}$ a finite range integral representation of $\mathbb{E}\left[P_{n, k_{1}}^{4}\right]$ is readily obtained [15] using the Q-function representation of [13]. This is easily evaluated.

Finally, the correlation between the subcarrier average error rate and subcarrier average squared error rate is given by

$$
\mathbb{E}\left[P_{n, a v} P_{n, a v}^{2}\right]=\frac{1}{N^{2}} \sum_{k_{1}=1}^{N} \sum_{k_{2}=1}^{N} \mathbb{E}\left[P_{k_{1}}^{2} P_{k_{2}}\right] .
$$

An expression for $\mathbb{E}\left[P_{n, k_{1}} P_{n, k_{2}}^{2}\right]$ is given in [15] and can be used to compute (36), specifically

$$
\mathbb{E}\left[P_{k_{1}}^{2} P_{k_{2}}\right]=\sum_{i=0}^{\infty} \frac{\theta^{2 i}}{4^{i} \beta^{i+1}} \kappa C_{i} D_{i} .
$$

In the special case of $k_{1}=k_{2}$ a finite range integral representation of $\mathbb{E}\left[P_{n, k_{1}}^{3}\right]$ is readily obtained [15] using the Q-function representation of [13]. This is easily evaluated.

\section{REFERENCES}

[1] T. Keller and L. Hanzo, "Adaptive multicarrier modulation: A convenient framework for time-frequency processing in wireless communications," Proc. IEEE, no. 5, pp. 611-640, May 2000.

[2] A. Barbour and P. Hall, "On the rate of Poisson convergence," Mathematical Proceedings of the Cambridge Philosophical Society, vol. 95, no. 3, pp. $473-480,1984$.

[3] A. R. S. Bahai and B. R. Saltzberg, Multi-Carrier Digital Communications. New York, NY: Kluwer Academic, 1999.

[4] W. C. Jakes, Microwave Mobile Communications. New York, NY: IEEE Press, 1994.

[5] M. A. Arcones, "Limit theorems for nonlinear functionals of a stationary Gaussian sequence of vectors," The Annals of Probability, vol. 22, no. 4, pp. 2242-2274, 1994.

[6] J. G. Proakis, Digital Communications, 4th ed. New York, NY: McGraw-Hill, 2001.

[7] Digital Video Broadcasting (DVB-T); Framing structure, channel coding, and modulation for digital terrestrial television, ETSI Std. ETS 300744 , Dec. 2001.

[8] I. Borisov and P. Ruzankin, "Poisson approximation for expectations of unbounded functions of independent random variables," The Annals of Probability, vol. 30, no. 4, pp. 1657-1680, 2002.

[9] K. Choi and A. Xia, "Approximating the number of successes in independent trials: Binomial versus Poisson," The Annals of Probability, vol. 12, no. 4, pp. 1139-1148, 2002.

[10] J. Hodges and L. Le Cam, "The Poisson approximation to the Poisson binomial distribution," The Annals of Mathematical Statistics, vol. 31, no. 3, pp. 737-740, 1960.

[11] L. H. Chen, "On the convergence of Poisson binomial to Poisson distributions," The Annals of Probability, vol. 2, no. 1, pp. 178-180, 1974.

[12] G. Marsaglia, "Ratios of normal variables and ratios of sums of uniform variables," J. Am. Stat. Assoc., vol. 60, no. 309, pp. 193-204, Mar. 1965.

[13] J. Craig, "A new, simple and exact result for calculating the probability of error for two-dimensional signal constellations," in Proc. IEEE MILCOM'91 Conf. Rec., Boston, 1991, pp. 25.5.1-25.5.5.

[14] R. K. Mallik, "On multivariate Rayleigh and exponential distributions," IEEE Trans. Inform. Theory, vol. 49, no. 6, pp. 1499-1515, June 2003.

[15] A. Clark. "Approximating the probability distribution of OFDM symbol errors," Technical Report, University of Canterbury, Christchurch, New Zealand, Mar. 2005. [Online]. Available: www.elec.canterbury.ac.nz/research/communications

[16] I. S. Gradshteyn and I. M. Ryzhik, Table of Integrals, Series, and Products. New York, NY: Academic Press, 1965. 